\title{
Transient Elastography for Assessment of Non-Alcoholic Fatty Liver Disease
}

\author{
Ludovico Abenavoli \\ University "Magna Græcia" of Catanzaro,
}

Italy

\section{Introduction}

In 1958, Menghini published the first description of liver biopsy (LB) as it pertained to the study of fibrosis. Since then, LB has become the "gold standard" in the evaluation of liver fibrosis. However, the use of LB has several limitations, including physical and mental discomfort of patients, which may lead to a high percentage of refusals, non-negligible morbidity and occasional mortality. Fibrosis is evaluated by histological semiquantitative scores, among which the METAVIR fibrosis scoring system is the most used; it is able to detect different degrees of fibrosis, from absence of fibrosis (F0) to cirrhosis (F4), and shows a better intra- and inter-observer reproducibility than other scales (i.e., Brunt, Knodell, Ishak and Scheuer). However these scores do not provide a dynamic picture of the disease, but only information about diagnosis and prognosis, such as the necroinflammatory activity and the presence of steatosis (Abenavoli et al, 2007).

Non-alcoholic fatty liver disease (NAFLD) is a relevant issue in public health because of its epidemiologic burden (Argo \& Caldwell, 2009). NAFLD is also not a new condition, but was not appreciated in early reports. It represents the most common chronic liver disease in the general population and is expected to increase in the future as a result of an ageing population, the improving control of other major causes of chronic liver disease and the epidemics of obesity and diabetes (Abenavoli et al, 2011). Steatosis consists of an accumulation of fat in the liver cells, and is not a disease in and of itself. It is an aggravating factor in certain pathological situations of varying degrees of gravity, and becomes dangerous when associated with inflammation or fibrosis. It is reversible, however, through diet and exercise. In fact NAFLD, may present as a spectrum ranging from asymptomatic steatosis with elevated or normal aminotransferases to steatohepatitis [non-alcoholic steatohepatitis (NASH)], all the way to cirrhosis with complications of liver function to hepatocellular carcinoma. NAFLD occurs in $60-95 \%$ patients with obesity, in $28-55 \%$ patients with type 2 diabetes mellitus (T2DM) and in $27-92 \%$ patients with dyslipidemia. Insulin resistance with compensatory hyperinsulinemia is the common denominator of obesity, T2DM and dyslipidemia and it can play a pathogenetic role in NAFLD. Accordingly, it has been reported that insulin resistance is the single laboratory finding most closely associated with NAFLD in a large series of patients, irrespective of body mass index (BMI), fat distribution, or glucose tolerance. Indeed, NAFLD has been recently proposed as an additional feature of the metabolic syndrome.

Currently liver steatosis, can be detected only by ultrasound (US) and quantified only by performing a biopsy. LB is still considered the "gold standard" for distinguishing between the 
broad range of NAFLD. However LB is not recommended in NAFLD patients, because of its cost, the potential risk of bleeding, and the absence of consensus regarding the histopatological criteria that firmly define NASH and differentiate between NAFLD entities. Due to the remarkable increase in the prevalence of NAFLD and the concomitant efforts in developing new therapies for fatty liver, non-invasive, simple, reproducible, and reliable non-invasive techniques are needed. In this way US is widely used in the diagnosis of patients with suspected liver disease because it is highly accurate, relatively inexpensive and non-invasive. Based on these characteristics, US has been the first-line imaging technique suitable both in the clinical setting and for epidemiological studies. In particular US is the least expensive modality for detecting changes associated with NAFLD. However, echography-based US techniques begin to become sensitive at moderately high levels of steatosis ( $33 \%$ or more) and suffer from low intra- and inter-operator repeatability (Schwenzer et al, 2009).

Vibration-controlled transient elastography ([VCTE], Fibroscan ${ }^{\circledR}$, Echosens, Paris, France) is a non-invasive technique which measures liver stiffness, and allows the assessment of the extent of hepatic fibrosis. VCTE has been proposed for the measurement of liver stiffness, considered to be one of the direct consequences of the fibrotic evolution of chronic liver disease (Sandrin et al, 2003).

A few meta-analyses have suggested liver stiffness measurement (LSM) through VCTE to be a reliable tool to detect advanced liver fibrosis and early liver cirrhosis (Friedrich-Rust et al, 2008). In NAFLD patients, various degrees of hepatic steatosis may attenuate the elastic shear wave, but it does not change its underlying speed which is the parameter used for stiffness measurement. Literature showed a clearly positive correlation between liver stiffness and the severity of liver fibrosis in patients with NAFLD. Rapid, non-invasive estimation of the stage of fibrosis in NAFLD patients, especially NASH patients, is of major clinical interest because such patients have been shown to be at a high risk of developing complications. Recently, a new measurement technique, the controlled attenuation parameter (CAP®, EchoSens, Paris, France), which provides good accuracy with even low levels of steatosis in the liver has been introduced. Because of its sensitivity at early phases of steatosis. CAP could provide a means to monitor the progression or regression of steatosis.

The combination of the measurement of liver attenuation parameter (CAP) and liver stiffness (VCTE) could provide a means to follow NAFLD patients throughout the course of the disease and observe independently the effects of steatosis and liver injury effects that are signs of the worsening of the disease.

\section{Transient elastography for staging liver disease}

The development of non-invasive tools to evaluate liver disease stages, has emerged as a major clinical and research priority. The quest for accurate techniques to stage NAFLD stems from a variety of recent data. First, the prevalence of NAFLD has grown to epidemic proportions. It is currently one of the most common chronic liver diseases worldwide. It is strongly associated with metabolic syndrome and obesity, and may progress to cirrhosis and hepatocellular carcinoma. The prognosis depends heavily on histological severity. Although patients with simple steatosis have excellent prognosis, those with NASH tend to progress and have hepatic complications. In Western countries, one in three men and nearly every other woman is affected by NAFLD. Second, it is well reported that NAFLD exists as a spectrum consisting of two major phenotypes that have drastically different natural histories. The majority of patients present simple steatosis, which has a benign clinical 
course. However $10-20 \%$ of these individuals present NASH that can evolve into cirrhosis. Third, according to literature, several problems for the management of NAFLD patients in clinical practice still exist: how to diagnose NAFLD and its type, how to select patient candidates for treatment and how to treat the patients selected. Discovery and validation of new diagnostic tools for NAFLD have several potential benefits, including reduced risk, reduced cost, as well as insights into disease mechanisms and pathogenesis. More accurate detection and diagnosis of NAFLD will help focus service provision for what is already a very common condition.

The limitations of LB (invasive procedure, sampling errors, interobserver variability and nondynamic evaluation) have stimulated the search for non-invasive approaches for the assessment of steatosis and liver fibrosis in patients with NAFLD. A variety of new methods, including serum markers, imaging techniques such as US, computed tomography, magnetic resonance imaging and VCTE, and more recently, CAP, have been proposed. In particular VCTE has shown excellent results for the diagnosis of severe fibrosis and cirrhosis and moderate results for the diagnosis of significant fibrosis in patients with NAFLD/NASH.

The stiffness of a body is defined as the ability of the body to deform itself under the action of a mechanical force (Abenavoli et al, 2007). The stiffness of a soft tissue can be estimated on the basis of the speed of propagation of a transverse shear elastic wave. The higher is the speed of propagation of that wave, the higher is the stiffness of the tissue. VCTE measures such speed of propagation in relatively homogenous organs like the liver, by using US pulses to localise the shear elastic wave at different times. The measuring device is equipped with a probe consisting of an ultrasonic transducer mounted on the axis of a vibrator. A low-frequency at $50 \mathrm{~Hz}$ and mild amplitude vibration, referred to as "shots", is transmitted from the vibrator to the tissue by the transducer itself. This vibration induces an elastic shear wave which propagates through the tissue. In the meantime, ultrasonic acquisitions are performed by an echographic transducer, using a frequency at $3.5 \mathrm{MHz}$, to determine the propagation of the shear wave. The propagation speed is transformed into stiffness by a formula derived by the physics of the propagation of a transverse shear elastic wave of a given frequency $(50 \mathrm{~Hz})$ through the soft tissue and expressed in kiloPascals $(\mathrm{kPa})$. The probe is applied perpendicularly to the skin (with a little gel film) through one of the right side intercostal spaces along the mid-axillary line. The measurement of the speed is taken along a cylinder of tissue ranging from 25 to 65 millimetres of depth under the skin. This corresponds to a volume of liver tissue approximately 100 times greater than an LB specimen and represents about $1 \%$ of the total organ volume. The examination is noninvasive and can be performed on ambulatory patients, in an outpatient setting or at the bedside of a hospitalized patient. VCTE can be performed indifferently by hepatologists or medical staff (physician, resident, medicine student, nurse) after a single training session provided by a specifically certified trainer. Results of the measurements range from 1.3 and $75.4 \mathrm{kPa}$. The manufacturer currently recommends that fibrosis score should be established from the median value of at least 10 successful acquisitions, with the rate of valid measures always higher than $50 \%$. The intra- and inter-observer coefficients of variation are 3.2 and $3.3 \%$, respectively, indicating very good reproducibility and operator independence.

\section{Elastography assessment of fatty liver}

A prospective study (Kim et al., 2007) aimed to assess the ability of VCTE to identify histologic parameters, including steatosis, in asymptomatic healthy individuals such as 
potential liver donors, and to compare these findings with results in liver disease patients. Forty-seven patients with abnormal liver function and/or hepatitis symptoms and 80 living related potential liver donors were consecutively enrolled, and LB and a LSM was performed in each subject. Histologic parameters were evaluated according to METAVIR score by a single pathologist. In liver disease patients, stiffness was significantly correlated with fibrosis stage $(P<0.001)$, and the optimal stiffness cut-off values for $\mathrm{F} \geq 2, \mathrm{~F} \geq 3$, and $\mathrm{F}=4$ were $7.35,8.85$, and $15.1 \mathrm{kPa}$ respectively. In potential liver donors, stiffness was not correlated with fibrosis $(P=0.851)$. In the latter group, the area under the receiver-operating characteristics curve (AUROC) was 0.70 (95\%, confidence interval [CI], 0.58-0.81), and the optimal stiffness cut-off value was $4.00 \mathrm{kPa}$ for $\mathrm{F} \geq 2$, which was lower than that in liver disease patients. Steatosis was not correlated with stiffness $(P=0.463)$ in potential liver donors. The Authors concluded that VCTE presets limited value for detecting steatosis in asymptomatic healthy individuals.

Nobili et al. (2008) have been assessed the value of VCTE in a cohort of pediatric patients with NASH. TE was performed in 50 consecutive biopsy-proven NASH patients age 13.6 \pm 2.44 years). The AUROC for the prediction of different degrees of liver fibrosis on the basis of Brunt score, were 0.977, 0.992, and 1, for respectively "any", significant and advanced fibrosis. Calculation of multilevel likelihood ratios shows that VCTE values $<5$, $<7$, and $<9 \mathrm{kPa}$, suggest the presence of "any" fibrosis, significant fibrosis, and advanced fibrosis, respectively. VCTE values between 5 and $7 \mathrm{kPa}$ predict a fibrosis stage of 1 , but with some degree of uncertainty. VCTE values between 7 and $9 \mathrm{kPa}$ predict fibrosis stages 1 or 2, but cannot discriminate between these two stages. VCTE values of at least $9 \mathrm{kPa}$ are associated with the presence of advanced fibrosis. In summary this study reported the reliability of VCTE to predict the presence of "any" fibrosis, significant fibrosis, and advanced fibrosis in these special populations.

Yoneda et al. (2008) have been investigated the usefulness of LSM in the evaluation of liver fibrosis in NAFLD patients. VCTE was performed for liver stiffness measurement in 97 NAFLD patients. The Authors have also investigated the relationship between LSM and the serum levels of hyaluronic acid and type IV collagen $7 \mathrm{~s}$ domain. Liver stiffness was well correlated with the different stage of METAVIR score $(P<0.0001)$. The AUROC were 0.927 for $\geq F 1,0.865$ for $\geq F 2,0.904$ for $\geq F 3,0.991$ for $\geq F 4$. Only fibrosis stage was strictly correlated with liver stiffness measurement by multiple regression analysis. Liver stiffness was also strongly correlated with the serum levels of type IV collagen $7 \mathrm{~s}$ domain $(\mathrm{r}=0.525, P<0.0001)$ and hyaluronic acid $(\mathrm{r}=0.457, P<0.0001)$. These data show a significant correlation between liver stiffness measurement and fibrosis stage in NAFLD patients, as confirmed by the results of LB.

Lupsor et al. (2010) assess VCTE performance in NASH patients, as well as the factors determining the discordance between the VCTE-predicted and the fibrosis stage evaluated by the Brunt system. LB and VCTE were performed on 72 consecutive NASH patients. LSM ranged from 2.80 to $16.90 \mathrm{kPa}$. In the univariate analysis, LSM was correlated with fibrosis $(P<0.0001)$, steatosis $(P<0.0001)$, ballooning $(P=0.001)$ and lobular inflammation $(P=0.002)$. In multivariate analysis, only fibrosis significantly correlated with LS $(P<0.0001)$. The median LSM values according to the fibrosis stages were: $4.90 \mathrm{kPa}$ (range: $2.80-7.30$ ) for F0; $6.15 \mathrm{kPa}$ (4.80-12.50) for F1; $6.90 \mathrm{kPa}(3.30-16.90)$ for F2 and $14.00 \mathrm{kPa}(10.70-14.10)$ for F3, with significant difference between stages, except for F1-F2 $(P=0.249)$. Cut-off values were calculated for predicting each fibrosis stage: $5.3 \mathrm{kPa}(\mathrm{AUROC}=0.879$ ) for $\mathrm{F} 1 ; 6.8 \mathrm{kPa}$ $(\mathrm{AUROC}=0.789)$ for F2; and $10.4 \mathrm{kPa}(\mathrm{AUROC}=0.978)$ for F3. Patients with false positive 
results had a significantly higher ALT level than those with concordant results $(P=0.039)$. These data show that steatosis degree, ballooning and inflammation do not influence LSM. In NAFLD patients, various degrees of hepatic steatosis may attenuate the elastic shear wave, possibly leading again to an underestimation of liver damage. Wong et al. (2010) aimed to evaluate the accuracy of VCTE in the diagnosis of fibrosis and cirrhosis in patients with NAFLD and to study factors associated with discordance between LSM and histology. Two hundred forty-six consecutive patients from two ethnic groups had successful LSM and satisfactory liver biopsy specimens. The AUROC of VCTE for F3 or higher and F4 disease was 0.93 and 0.95 , respectively, and was significantly higher than that of other prediction scores

(e.g. aspartate aminotransferase-to-alanine aminotransferase ratio, aspartate aminotransferase-to-platelet ratio index, FIB-4, BARD, and NAFLD fibrosis scores). AUROC ranged from 0.62 to $0.81, P<0.05$ for all comparisons. At a cut-off value of $7.9 \mathrm{kPa}$, the sensitivity, specificity, and positive and negative predictive values for F3 or greater disease were $91 \%, 75 \%, 52 \%$, and $97 \%$, respectively. LSM was not affected by hepatic steatosis, necroinflammation, or body mass index. Discordance of at least two stages between VCTE and histology was observed in $33(13.4 \%)$ patients. By multivariate analysis, a LB length less than $20 \mathrm{~mm}$ and F0-2 disease, rather than VCTE, were associated with discordance. Unsatisfactory LB specimens rather than VCTE account for most cases of discordance. However, when the diagnostic characteristics were compared using an "intention to diagnose" approach with the inclusion of subjects who had unsuccessful TE acquisition, the sensitivity and specificity values were not dissimilar from the clinical/biochemical models, although 95\% CIs were not provided for statistical comparison.

Subsequently a prospective study by Gaia et al. (2011) assess the accuracy and the efficacy of VCTE for the detection of fibrosis in patients with liver disease of different etiology (chronic hepatitis B - CHB, C- CHC and NAFLD) and evaluate the effect of steatosis on LSM. VCTE was performed in 219 consecutive patients with chronic liver disease (35\% CHC, 32\% CHB, and 33\% NAFLD) within 6 months of the liver biopsy. LSM was related to the fibrosis stage in each group (CHC: $P=0.596, P<0.001$; $C H B: P=0.418, P<0.001$; NAFLD: $P=0.573, P<0.001$ ), but the correlation was less strong in CHB and NAFLD than in CHC patients. Median LSM values were $7 \mathrm{kPa}(3.2-26)$ in $\mathrm{HCV}$ patients $(\mathrm{n}=77), 7.6 \mathrm{kPa}(3.7-30.7)$ in $\mathrm{HBV}$ patients $(\mathrm{n}=70)$, $6.6 \mathrm{kPa}(3.0-44.3)$ in NAFLD patients $(\mathrm{n}=72)$ compared to $4.35 \mathrm{kPa}$ (range $2.6-7)$ in controls $(n=40),(P<0.001)$. The median values of LSM according to fibrosis stage evaluated by METAVIR were in the 72 NAFLD patients: F0: $5.3 \mathrm{kPa}$ (3.0-9.7), F1: $6.15 \mathrm{kPa}(3.2-12.1)$, F2:7.75 $\mathrm{kPa}(4.3-13.9), \quad \mathrm{F} 3: 6.5 \mathrm{kPa}(4.3-10.3), \quad \mathrm{F} 4: 11.9 \mathrm{kPa}$ (7.9-44.3), $(P=0.001)$. VCTE underestimated the stage of fibrosis in $75 \%$ of patients with $\mathrm{F} 3$ and steatosis $>33 \%$. At multiple logistic regression analysis, in CHC and CHB patients, LSM was the only predictive variable of severe fibrosis/cirrhosis (odds ratio $[\mathrm{OR}]=1.42, P=0.003$ and $\mathrm{OR}=1.354$, $P=0.003$, respectively), while in NAFLD subjects BMI and AST (OR=1.433, $P=0.002$ and $\mathrm{OR}=1.053, P=0.020$, respectively) but not LSM were independently related with advanced fibrosis and cirrhosis. This study confirms that VCTE can be considered a valid support to detect fibrosis in chronic liver disease related to HCV but it should be interpreted cautiously in $\mathrm{CHB}$ and NAFLD patients.

\section{Elastography assessment of NAFLD in obese patients}

LSM can be influenced by metabolic syndrome even in the absence of biological features in NAFLD. It would be important to identify the rates and factors associated with unreliable 
LSM or LSM failure, as the results may provide guidance to referral physicians regarding the optimal candidates for LSM. The knowledge would also assist clinicians to counsel patients before arranging LSM. However, studies have shown that BMI>28 is an independent risk factor for a LSM failure (Foucher et al, 2006). In a prospective pilot study on VCTE in NAFLD, de Lédinghen et al. (2010) aimed to assess the feasibility of LSM when using a new XL-probe on patients with a $B M I \geq 30 \mathrm{~kg} / \mathrm{m}^{2}$ is used. In this study 99 patients (mean BMI $40.5 \mathrm{~kg} / \mathrm{m}^{2}$ ) were included. LSM was successful in $45 \%$ of the cases with the M probe, versus $76 \%$ of the cases with the XL-probe $(P<0.001)$. Fifty-nine percent of those who could not be measured ( $<10$ valid measurements) using the M-probe could successfully be measured using the XL-probe. In the 44 patients successfully measured with both probes, LSM was correlated with the platelet count, prothrombin time, gamma-glutamyltransferase, aspartate aminotransferase, fasting glucose, AST platelet ratio index, Forns score and FIB-4. The XL-probe allows providing a higher rate of LSM than the M-probe in patients with an increased BMI and shows promising results for the evaluation of liver fibrosis.

\begin{tabular}{|c|c|c|c|c|}
\hline Lead author & Yoneda $(N$ 97)* & Nobili $(N 50)^{\circ}$ & Lupsor $(N 72)^{\circ}$ & Wong (N 246) ${ }^{\circ}$ \\
\hline \multicolumn{5}{|l|}{$F \geq 2$} \\
\hline AUROC & 0.86 & 0.99 & 0.78 & 0.84 \\
\hline Optimal cut-off (kPa) & 6.6 & 7.4 & 6.8 & 7.0 \\
\hline Sensitivity (\%) & 88 & 100 & 100 & 79 \\
\hline Specificity (\%) & 74 & 92 & 97 & 76 \\
\hline PPV (\%) & 79 & 80 & 71 & 70 \\
\hline NPV (\%) & 85 & 100 & 100 & 84 \\
\hline \multicolumn{5}{|l|}{$F \geq 3$} \\
\hline AUROC & 0.90 & 1.0 & 0.978 & 0.93 \\
\hline Optimal cut-off $(\mathrm{kPa})$ & 9.8 & 10.2 & 10.4 & 8.7 \\
\hline Sensitivity (\%) & 85 & 100 & 100 & 84 \\
\hline Specificity (\%) & 81 & 100 & 97 & 83 \\
\hline PPV (\%) & 64 & 100 & 71 & 59 \\
\hline NPV (\%) & 93 & 100 & 100 & 95 \\
\hline \multicolumn{5}{|l|}{$F \geq 3$} \\
\hline AUROC & 0.99 & - & - & 0.95 \\
\hline Optimal cut-off $(\mathrm{kPa})$ & 17.5 & & & 10.3 \\
\hline Sensitivity (\%) & 100 & - & - & 92 \\
\hline Specificity (\%) & 97 & - & - & 88 \\
\hline PPV (\%) & 75 & - & - & 46 \\
\hline NPV (\%) & 100 & - & - & 99 \\
\hline
\end{tabular}

Table 1. Analysis of VCTE cut-off for the diagnosis of NAFLD in adult patients. (AUROC: area under receiver operative curve; PPV: positive predictive value; NPV: negative predictive value. Histological evaluation by *METAVIR and ${ }^{\circ}$ Brunt score).

In this way Wong et al. (2011) have recently investigated the rates of unreliable LSM and LSM failure in patients suffering from chronic liver diseases. They also aimed to evaluate the factors including BMI and central obesity associated with unreliable LSM and LSM failure. Among 3205 Chinese patients with LSM, 370 (12\%) with liver steatosis, 371 $(11.6 \%)$ and $88(2.7 \%)$ had unreliable LSM and LSM failure, respectively. The rates started 
to increase when $\mathrm{BMI} \geq 28.0 \mathrm{~kg} / \mathrm{m}^{2}$. Comparing patients with $\mathrm{BMI} \geq 28.0-29.9 \mathrm{~kg} / \mathrm{m}^{2} \mathrm{vs}$ those with $\mathrm{BMI} \geq 30.0 \mathrm{~kg} / \mathrm{m}^{2}$, the rates of unreliable LSM $(16.4 \%$ vs $18.9 \% ; P=0.62)$ and LSM failure $(11.8 \%$ vs $17.8 \% ; P=0.16)$ were similar. $B M I \geq 28.0 \mathrm{~kg} / \mathrm{m} 2$ was the most important factor associated with unreliable LSM (OR=2.9, 95\% CI=2.1-3.9, $P<0.0001)$ and LSM failure $(\mathrm{OR}=10.1, \quad 95 \% \quad \mathrm{CI}=6.4-14.2, \quad P<0.0001)$. Central obesity, defined as waist circumference $>80 \mathrm{~cm}$ in women and $>90 \mathrm{~cm}$ in men, was another independent risk factor of unreliable LSM (OR=1.3, 95\% CI=1.0-1.6, $P=0.04)$ and LSM failure $(\mathrm{OR}=5.8,95 \%$ $\mathrm{CI}=2.9-11.5, P<0.0001)$.

\section{Controlled attenuation parameter and liver steatosis}

Fat affects US propagation, however this is the most common liver imaging technique used to detect the presence of steatosis. In B-mode images, steatosis appears as an increased parenchymal echogenicity caused by the increased reflectivity induced by fatty accumulation. This increased echogenicity is determined by the sonographer, most of the time by comparison with kidney echogenicity. Such as procedure it is highly operatorand machine-dependant. Many studies have reported averaged specificity and sensitivity of the technique as around $60-70 \%$. Other studies have reported better specificity and sensitivity up to $95 \%$, and therefore its performance in clinical practice is still controversial. Another limitation of US is that it cannot quantify steatosis and can only detect steatosis from around $30 \%$ of fatty infiltration, where there is a clinical relevance to detect lower extent of liver fat.

Furthermore, some studies have shown that liver fibrosis can induce an increased liver echogenicity without posterior beam attenuation. It is therefore impossible to distinguish between increased echogenicity caused by an extensive fibrosis from increased echogenicity caused by fatty infiltration. A quantitative attenuation parameter can overcome this limitation.

Recently a new parameter has been developed to detect and quantify degree of liver steatosis. This parameter is based on the US properties of the radiofrequency backpropagated signals acquired by the VCTE. It is called controlled attenuation parameter (CAP), because it was devised to specifically target the liver (Sasso et al, 2010). This coefficient is an estimate of the total US (go-and-return path) at $3.5 \mathrm{MHz}$ and is expressed in decibel per meters $\left(\mathrm{dB} \cdot \mathrm{m}^{-1}\right)$. CAP is evaluated using the same radiofrequency data and in the same region of interest than the ones used for LSM and it is only appraised if the acquisition is valid. CAP is VCTE guided and obtains an ultrasonic attenuation value of the liver only. Therefore, CAP can be assessed by an operator who does not have any ultrasound imaging skills. Furthermore, CAP has been designed to be immediate, reproducible and operator and machine-independent. Performance of CAP was evaluated by Sasso et al. on 115 patients, taking the histological grade of steatosis as reference. Among those patients, 42 were $\mathrm{CHC}, 17 \mathrm{CBV}, 39$ with alcoholic liver disease and 17 with NAFLD. Mean BMI was $25 \pm 4 \mathrm{~kg} / \mathrm{m}^{2}$. CAP was significantly correlated to steatosis $\left(P=10^{-16}\right)$. AUROC was equal to 0.91 and 0.95 for the detection of more than $10 \%$ and $33 \%$ of steatosis, respectively. These data show that CAP can efficiently separate several steatosis grades. CAP appears to be a promising diagnostic tool for non-invasive assessment and quantification of steatosis, enhancing the spectrum of the non-invasive method for the exploration and follow-up of patients with fatty liver. However validation of this novel indicator is ongoing in a large multi-etiology cohort study. 


\section{Conclusion}

LB will still be part of clinical practice in the coming years, but progress in medicine will challenge previously entrenched assumptions and will change our current approach to liver diseases in the next future. Non-invasive assessment of liver fibrosis is currently a reality in patients with CHC. In other highly prevalent diseases, such as NAFLD there is still room for improvement, although in the next few years LB will likely be supplanted in most cases. Many imaging techniques can be used to identify liver steatosis, such as computed tomography scans, magnetic resonance imaging or proton nuclear magnetic resonance. However all of these imaging tools present some important limitations: they are not easily available and their costs are relatively expensive (Sasso et al, 2010).

VCTE is a simple, non-invasive and inexpensive method used to measure LS. The accuracy and reproducibility of VCTE score is excellent for the diagnosis of cirrhosis; it is probably the most accurate non-invasive method for the early detection of cirrhosis. It is a userfriendly technique that can be performed without any preparation in the less than five minutes in clinic or at the bedside, with immediate results and high patient acceptance, it is very likely that in future it will become the most widely used technique for staging liver disease. A panel discussion is in progress to define the role of VCTE in the staging of liver disease. In particular, there are a few methodological approaches that need to be implemented in future studies designed to further validate LSM. A new statistical approach is needed in order to obtain reliable and clinically useful information from LSM (Vizzuti et al, 2009). In fact, most of the published studies reported the cut-off values selected by binary measures, like sensitivity and specificity. However, it seems insufficiently informative to discriminate the optimal cut-off value in a wide range of values, specially in a context of liver steatosis. A new parameter measuring the US attenuation could be interesting and might be related to steatosis. This indicator, called CAP, has been developed to process the raw ultrasonic signals stored in the VCTE examination file. Performance of CAP has to be validated into specific etiologic groups. In addiction influence of the steatosis topography and other histologic constituents, specific to each etiology has to be considered. Large cohorts are required to determine appropriate threshold to detect and quantify steatosis.

Further technological improvements are requested for better application of this technique in specific populations, such as patients with increased BMI, along with efforts to improve and standardize the procedure and adequate operator training.

\section{Acknowledgment}

We would like to thank Emanuela Libri for the linguistic revision.

\section{References}

Menghini G.(1958). One-second needle biopsy of the liver. Gastroenterology. Volume 35, pp. 190-199.

Argo C.K. \& Caldwell S.H. (2009). Epidemiology and natural history of non-alcoholic steatohepatitis. Clin Liver Disease. Volume 13, pp. 511-531

Abenavoli L., Corpechot C. \& Poupon R. (2007). Elastography in hepatology. Canadian Journal of Gastroenterology. Volume 21, No. 12, pp. 839-842 
Abenavoli L., Aviello G., Capasso R., Milic N. \& Capasso F. (2011). Milk thistle for nonalcoholic fatty liver disease treatment. Hepatitis Monthly. Volume 11, No.3, pp. 173177

Schwenzer N.F., Springer F., Schraml C., Stefan N., Machann J. \& Schick F. (2009). Noninvasive assessment and quantification of liver steatosis by ultrasound, computed tomography and magnetic resonance. Journal of Hepatology. Volume 51, No. 3, pp.433-445.

Sandrin L., Fourquet B., Hasquenoph J.M., Yon S., Fournier C., Mal F., Christidis C., Ziol M., Poulet B., Kazemi F., Beaugrand M. \& Palau R. (2003). Transient elastography: A new noninvasive method for assessment of hepatic fibrosis. Ultrasound in Medicine and Biology. Volume 29, No.11, pp.1705-1713.

Friedrich-Rust M., Ong M.F., Martens S., Sarrazin C., Bojunga J., Zeuzem S. \& Herrmann E. (2008). Performance of transient elastography for the staging of liver fibrosis: a meta-analysis. Gastroenterology. Volume 134, No. 4 pp. 960-974.

Kim K.M., Choi W.B., Park S.H., Yu E., Lee S.G., Lim Y.S., Lee H.C., Chung Y.H., Lee Y.S., \& Suh D.J. (2007). Diagnosis of hepatic steatosis and fibrosis by transient elastography in asymptomatic healthy individuals: a prospective study of living related potential liver donors. Journal of Gastroenterology. Volume 42, No. 5, pp. 382-388.

Nobili V., Vizzutti F., Arena U., Abraldes J.G., Marra F., Pietrobattista A., Fruhwirth R., Marcellini M. \& Pinzani M. (2008). Accuracy and reproducibility of transient elastography for the diagnosis of fibrosis in pediatric nonalcoholic steatohepatitis. Hepatology. Volume 48, No. 2, pp. 42-48.

Yoneda M., Yoneda M., Mawatari H., Fujita K., Endo H., Iida H., Nozaki Y., Yonemitsu K., Higurashi T., Takahashi H., Kobayashi N., Kirikoshi H., Abe Y., Inamori M., Kubota K., Saito S., Tamano M., Hiraishi H., Maeyama S., Yamaguchi N., Togo S. \& Nakajima A (2008). Noninvasive assessment of liver fibrosis by measurement of stiffness in patients with nonalcoholic fatty liver disease (NAFLD). Digestive and Liver Disease. Volume 40, No 5, pp. 371-378.

Lupsor M., Badea R., Stefanescu H., Grigorescu M., Serban A., Radu C., Crişan D., Sparchez Z., Iancu S. \& Maniu A. (2010). Performance of unidimensional transient elastography in staging non-alcoholic steatohepatitis. Journal of Gastrointestinal and Liver Diseases. Volume 19, No. 1, pp. 53-60.

Wong V.W., Vergniol J., Wong G.L., Foucher J., Chan H.L., Le Bail B., Choi P.C., Kowo M., Chan A.W., Merrouche W., Sung J.J. \& de Lédinghen V. (2010). Diagnosis of fibrosis and cirrhosis using liver stiffness measurement in nonalcoholic fatty liver disease. Hepatology. Volume 51, No 2, pp. 454-462.

Gaia S., Carenzi S., Barilli A.L., Bugianesi E., Smedile A., Brunello F., Marzano A. \& Rizzetto M. (2011). Reliability of transient elastography for the detection of fibrosis in nonalcoholic fatty liver disease and chronic viral hepatitis. Journal of Hepatology. Volume 54, No. 1, pp. 64-71.

Foucher J., Castéra L., Bernard P.H., Adhoute X., Laharie D., Bertet J., Couzigou P. \& de Lédinghen V. (2006). Prevalence and factors associated with failure of liver stiffness measurement using FibroScan in a prospective study of 2114 examinations. European Journal of Gastroenterology and Hepatology. Volume 18, No 4, pp. 411-412. 
de Lédinghen V., Vergniol J., Foucher J., El-Hajbi F., Merrouche W. \& Rigalleau V. (2010). Feasibility of liver transient elastography with FibroScan using a new probe for obese patients. Liver International. Volume 30, No. 7, pp. 1043-1048.

Wong G.L., Wong V.W., Chim A.M., Yiu K.K., Chu S.H., Li M.K. \& Chan H.L. (2011). Factors associated with unreliable liver stiffness measurement and its failure with transient elastography in the Chinese population. Journal of Gastroenterology and Hepatology. Volume 26, No. 2, pp. 300-305.

Sasso M., Beaugrand M., de Ledinghen V., Douvin C., Marcellin P., Poupon R., Sandrin L. \& Miette V. (2010). Controlled attenuation parameter (CAP): a novel VCTE ${ }^{\mathrm{TM}}$ guided ultrasonic attenuation measurement for the evaluation of hepatic steatosis: preliminary study and validation in a cohort of patients with chronic liver disease from various causes. Ultrasound in Medicine E Biology. Volume 36, No 11, pp.18251835.

Vizzutti F., Arena U., Nobili V., Tarquini R., Trappoliere M., Laffi G., Marra F. \& Pinzani M. (2009). Non-invasive assessment of fibrosis in non-alcoholic fatty liver disease. Annals of Hepatology. Volume 8, No. 2, pp. 89-94. 


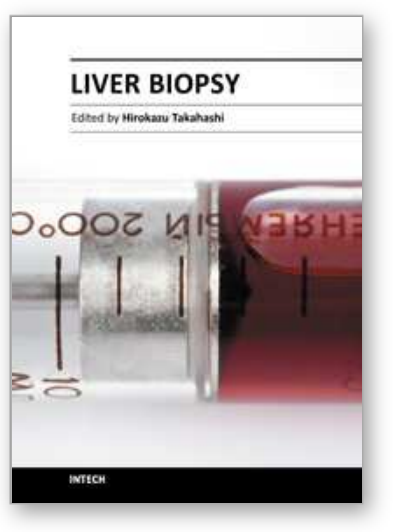

\author{
Liver Biopsy \\ Edited by Dr Hirokazu Takahashi
}

ISBN 978-953-307-644-7

Hard cover, 404 pages

Publisher InTech

Published online 06, September, 2011

Published in print edition September, 2011

Liver biopsy is recommended as the gold standard method to determine diagnosis, fibrosis staging, prognosis and therapeutic indications in patients with chronic liver disease. However, liver biopsy is an invasive procedure with a risk of complications which can be serious. This book provides the management of the complications in liver biopsy. Additionally, this book provides also the references for the new technology of liver biopsy including the non-invasive elastography, imaging methods and blood panels which could be the alternatives to liver biopsy. The non-invasive methods, especially the elastography, which is the new procedure in hot topics, which were frequently reported in these years. In this book, the professionals of elastography show the mechanism, availability and how to use this technology in a clinical field of elastography. The comprehension of elastography could be a great help for better dealing and for understanding of liver biopsy.

\title{
How to reference
}

In order to correctly reference this scholarly work, feel free to copy and paste the following:

Ludovico Abenavoli (2011). Transient Elastography for Assessment of Non-Alcoholic Fatty Liver Disease, Liver Biopsy, Dr Hirokazu Takahashi (Ed.), ISBN: 978-953-307-644-7, InTech, Available from: http://www.intechopen.com/books/liver-biopsy/transient-elastography-for-assessment-of-non-alcoholic-fattyliver-disease

\section{INTECH}

open science | open minds

\section{InTech Europe}

University Campus STeP Ri

Slavka Krautzeka 83/A

51000 Rijeka, Croatia

Phone: +385 (51) 770447

Fax: +385 (51) 686166

www.intechopen.com

\section{InTech China}

Unit 405, Office Block, Hotel Equatorial Shanghai

No.65, Yan An Road (West), Shanghai, 200040, China

中国上海市延安西路65号上海国际贵都大饭店办公楼 405 单元

Phone: +86-21-62489820

Fax: $+86-21-62489821$ 
(C) 2011 The Author(s). Licensee IntechOpen. This chapter is distributed under the terms of the Creative Commons Attribution-NonCommercialShareAlike-3.0 License, which permits use, distribution and reproduction for non-commercial purposes, provided the original is properly cited and derivative works building on this content are distributed under the same license. 